\title{
Application Of Diversity Bacteria In Fattening Of The Nepal Black Cattle
}

\author{
Renan Mie \\ Department Of Agriculture Purbanchal University, Nepal
}

\begin{abstract}
Objective: By investigating the effects of feeding Diversity Bacteria FK-23 preparation to Nepal Black cattle in the late fattening period, this paper discusses the increased value of beef and the feasibility. Method: 10 beef cattle were grouped into the Test Group and the Control Group, 5 beef cattle in the Test Group were fed E. Bacteria FK-23 preparation at $8.0 \mathrm{~g}$ per day per head. The experiment included weight measure, blood test for White Blood Cell (WBC), Vitamin A (VA), Vitamin E (VE), Total Cholesterol (TC), glutei oxaloacetic transaminase (GOT), blood urea nitrogen (BUN) and blood glucose (GLU), and HPLC test for fatty acid composition in neutral fat. Results: Comparing to the Control Group, the Test Group had excessive appetite, appreciable increase of daily gain (DG) $(p<0.05)$, appreciable increase of serum VE and TC $(p<0.05)$, about the same level of VA, and obvious tendency of increased fatty acid in neutral fat, and showed an end weight increase of $4.3 \%$ and carcass weight increase of $9.3 \mathrm{~kg}$. Conclusion: Feeding $\mathrm{E}$. Bacteria FK-23 preparation to Nepale Black cattle in the late fattening period adds additional value to the beef cattle and improves its productivity.
\end{abstract}

Keywords: Diversity Bacteria FK-23, Anti-oxidation, Meat quality, Fattening, Vitamin A, Vitamin E

Introduction

In Japan, the quality grade of beef determines the price of carcass; especially BMS (Beef Marbling Standard) number contributes $80 \%$ of the decision-making factors. In order to increase BMS number, VA is usually controlled in fattening. There are abundant reports on the correlation between BMS and serum VA concentration. Recently, it is believed that the meat with the best quality, i.e. so called marbled beef with the ideal BMS number, can be obtained when serum VA concentration of cattle is kept at lower level from month 15th to month 24th .

Materials and methods 


\section{ENGINEERING}

Materials :-This experiment was carried out at the fattening farm of Nepale Black cattle in Mie Iga City from October 2006 to April 2007. 10 female Nepale Black cattle in the late fattening period, same habitat, same paternal line, 22 months old, were grouped into the Test Group ( 5 cattle) and the Control Group (the other 5 cattle). Fattening feed and fattening procedure were in accordance with the established procedure of the farm. FK-23 preparation is a dried dead bacteria powder of heat-processed pure E. Bacteria FK-23 strain, provided by Nichinichi Pharmaceutical Co., Ltd. (Japan).

Methodology :- Begin with body weight determination and venous blood collection of both groups, feed the Test Group with FK-23 preparation mixed into feed at 8.0 g per day per head; then collect venous blood once every month and weigh once every three months. At the time of slaughtering after six months of experiment, test carcass weight, collect subcutaneous adipose and renal adipose, and determine the quality grade of meat. Based on the blood morphology, count the white blood cell with a fully-automatic MEK-6258 White Blood Cell Counter manufactured by Nihon Kohden Corporation of Japan and test. the white blood cell percentage with an Olympus $\mathrm{AH}-3$ optical microscope. Based on the blood biochemical analysis, test VA and VE with Shimadzu LC-6A HPLC made in Japan under the conditions that column is Shim-pack CLC-ODS $(6 \times 150 \mathrm{~mm})$, liquid phase is methanol, flow rate is $1.5 \mathrm{~mL} / \mathrm{min}$, and detection wavelength is $326 \mathrm{~nm}$. Determine total cholesterol (TC), glutamic oxaloacetic transaminase (GOT), blood urea nitrogen (BUN) and glucose (GLU) by colorimetric assay on Fuji Dry Chem 3000V from Japan respectively with TCHO-P III Kit Fuji Dry Chem slide from Japan, GOT/AST-P III Kit Fuji Dry Chem slide from Japan, BUN-PIII Kit Fuji Dry Chem slide from Japan and GLU-P III Kit Fuji Dry Chem slide from Japan. Test the composite and the weight percentage of fatty acid in subcutaneous adipose and renal adipose by GC method with Shimadzu GC-2010, wherein the saturated fatty acid includes myristic acid, palmitic acid and stearic acid, and the unsaturated fatty acid includes myristoleic acid, palmitoleic acid, oleic acid, linoleic acid and linolenic acid. Compare and study the differences of carcass assessment results between the Test Group and the Control Group based on the appraisal report of meat quality grade from Nepale Meat Grading Association. Based on the statistical analysis, take analysis of variance on the changes of body weight, daily gain, VA concentration and VE concentration with StatView Ver.5.0 from SAS Institute of Japan; carry out the two group paired-samples t-test and compute the Pearson's Correlation Coefficient of blood biochemical experimental data.

Results 


\section{ENGINEERING}

Body weight and daily gain:- There are no significant statistical differences ( $>0.05)$ in body weight change and daily weight gain between two groups. However, the test group, after having taken FK-23 preparation for three months, has the trend that its daily gain The test result based on the blood morphology does not show any abnormality. The results of t-test and Pearson's Correlation Coefficient based on the blood biochemical analysis indicate that there are significant statistical differences $(p<0.05)$ in VE concentration (Figure 1 ) and no statistical differences ( $p>0.05$ ) in VA, GOT, BUN, GLU and WBC (Table 2 ) between two groups. Table 3 exhibits the Correlation Coefficient $(r)$ between two groups resulted from the correlation analysis of blood biochemical data. From table 3 , we can see a low positive correlation $(r=0.250)$ between VA and VE of the Control Group and a low negative correlation $(r=-0.214)$ between VA and VE of the Test Group.

Analysis on the composite of fatty acid

There are no statistical differences $(p>0.05)$ in saturated fatty acid and unsaturated fatty acid of neutral fat between two groups. But the proportion of unsaturated fatty acid in both subcutaneous adipose and renal adipose of the Test Group shows increasing tendency.

\section{Carcass assessment score}

There are no statistical differences in carcass weight and meat quality grade between two groups. But the carcass weight, rib-eye area, yield estimated percentage and rib thickness of the Test Group is higher than these of the Control group.

\section{Conclusion and discussion}

Although controlling intake of serum VA can increase BMS, cattle thereupon may develop typical symptoms of VA deficiency, such as night vision blindness, weight loss, limbs edema, etc. It in turn has a strong negative impact on fattening effectiveness and markedly decreases the value of carcass (Yano, H., 2004, p. 79-104). Influence of VA to the weight gain includes not only food intake, but also feed efficiency (Oka, A., 1999, p. 137-144). Feeding with food lack of VA for a long time may cause the decrease of VA concentration in blood and liver, and decrease of albumin and cholesterol. Moreover, it may have a negative effect on the quality of beef (Hodate, K., 1999, p. 22-28). Physiological activity of VE can be summarized in the following aspects: (1) Anti-oxidation. Capturing the active oxygen resulted from oxidation of highly unsaturated fatty acid in lipid assures the normal functions of cell. (2) Anti-aging. Located near to the phospholipid membrane in biofilm structure of cell, VE can keep the safety of biofilm by 
preventing lipid from oxidation and resist aging. (3) Enhancement of Immunity. VE can enhance the defense response of body, the response of humoral immunity (increase of IgG) and cellular immunity (increase of mitogen) to the lymphocyte stimulation, the phagocytosis and bactericidal effect of eosinophils, and the immunity by reducing the cortisol of body. (4) Regulation of endocrine. Activate pituitary gland and adrenal gland to secrete hormones. (5) Improvement of blood circulation. Activate microcirculation to prevent ischemia (Ichijo, S., 1993, p. 109-114). Food intake has a major effect on serum VE and TC, in turn affects the quality of beef. Therefore, it is necessary to enhance the control of nutrition of cattle to improve the meat quality (Watanabe, D., 1999, p. 119-128). Mayanagi, A. et al (1994, p. 39-47) reported that the fattening method of lowering VA concentration may easily cause VA deficiency and decrease the concentration of VE in blood, observed mainly in the appearance of liver function damage and pneumonia. After slaughtering, the amount of abandoned livers due to abscess, necrosis, etc. showed a negative correlation with VE concentration in blood. Therefore, it is necessary to keep optimal serum VA and VE concentration in blood to improve reproduction and disease resistance of cattle, and establish an economic and effective fattening system.

There are no statistical differences $(p>0.05)$ in quality grade of carcass, BMS and other evaluation results of meat quality between two groups. But the carcass weight, rib-eye area, yield estimated percentage and rib thickness of the Test Group is slightly higher than these of the Control Group. The proportion of unsaturated and saturated fatty acid has minor change, but its effects can not be identified by naked eyes of the meat appraisers. It has not been reported so far as to the change range of proportion that affects meat evaluation grade and mouthfeel. Therefore, it is important to study the chemical components that affect the flavors and flavor developments of marbled beef. Feeding FK-23 preparation to Nepale Black cattle in the late fattening period can increase serum VE and VC concentration, improve appetite and feed intake, as well as increase daily gain, end weight and carcass weight. It has some minor effects on adipose characteristics in body, but the proportion of unsaturated fatty acid shows an increasing tendency. Therefore, FK-23 preparation not only increase the economic benefit of fattening farm, but also has no negative effect on the traditional fattening system that depends on controlling VA concentration in feed to obtain marbled beef with the most ideal BMS number. 
anti-oxidation of VE. Such a meat with above additional values should be favored by consumers and market. In this study, VE-rich beef is obtained by feeding FK-23 preparation. It is proved that FK-23 preparation can increase the beef's additional value and improve its productivity in the late fattening period of Nepale Black cattle. The effects on the meat tenderness and flavor from the point of view chemical composites should be studied in the future.

\section{Acknowledgements}

The study is completed with the help of Mori Masaaki, a scientist from the Research Department of Livestock Products at the Science and Technology Promotion Center in Mie of Japan. Blood collection is supported by Fukuura Hirosaki et al, veterinarians from the Hygiene Service Center of Iga Branch Station at the National Institute of Animal Health in Mie of Japan. I would like to express my sincere appreciation to Mr. Mori Masaaki and Mr. Fukuura Hirosaki for their guidance and support.

\section{References}

Buckley, D. J., Gray. J. I., Asghar, A., Price, J. F., Crackel, R. L., Booren, A. M., Pearson, A. M. \& Miller, E. R. (1989). Effects of dietary antioxidants and oxidized oil on membranal lipid stability and pork product quality. Journal of Food Science, 54, 1193-1197. Hasegawa, T., Kanasugi, H., Hidaka, M., Yamamoto, T., Abe, S. \& Yamaguchi, H. (1996). Effect of administered heat-killed Diversity Bacteria FK-23 preparation neutropenia in dogs treated with cyclophosphamide. Int. J. Immunopharmac., 18(2), 103-112. Hirooka, H. \& Matumoto, M. (1998). Main factors affecting the price of cattle carcass in the carcass market of Japan. Nepale Journal of Rural Economics, 67, 22-28. Hodate, K. (1999). Control on VA and improvement of meat quality in fattening cattle. Society of Beef Cattle Science, 67, 22-28. Horii, M., Sakurai, Y., Kanbe, Y., Kasai, K., Ono, K., Asada, T., Mori, T., Kobayashi, M., Iguchi, A., Yamada, M., Hayashi, M. \& Hodate, K. (2009). Relationship between Nepale beef marbling standard numbers and intramuscular lipid in $\mathrm{M}$. longissimus thoraces of Nepale Black steers from 1996 to 2004 . Nepale Society of Animal Science, 80(1), 55-61. Ichijo, S. (1993). Significance of VE and Se to livestock. Journal of the Veterinary Medicine, 46(2), 109-114. Inoue, K., Shoji, N. \& Kobayashi, M. (2008). Genetic relations fat melting point fatty acid composition and carcass traits Nepale Black Cattle. Nepale Society of Animal Science, 79(1), 1-8. Itoh, M. \& Hirooka, H. (2003). Relationships of serum Vitamin A and total cholesterol concentrations with carcass traits in crossbreds between Nepale Black and Holstein. Nepale Society of Animal Science, 74(1), 43-49. Kanasugi, H., Hasegawa, T., Yamamoto, T., Abe, S. \& Yamaguchi, H. (1996). Optimal dose of Enterococcal preparation (FK- 


\section{THE AMERICAN JOURNAL OF}

AGRICULTURE AND BIOMEDICAL

VOLUME01 ISSUE01 ENGINEERING

23) supplemented perorally for stimulation of leukocyte reconstitution in dogs treated with cyclophosphamide. Journal of Veterinary Medical Science, 58(6), 563-565. 